\title{
Injerto de Piel de Espesor Total en Recurrencia de Dupuytren: Resultados Tempranos
}

\section{Skin Graft for Recurrent Dupuytren Disease: Early Results}

\author{
Raquel A. Montes-Pérez ${ }^{1,2}$ Miguel Del Cerro-Gutiérrez ${ }^{3,4} \quad$ Francisco García-Lira $^{5}$
}

\footnotetext{
${ }^{1}$ Cirujana Traumatólogo Ortopedista, Fellowship en Cirugía de la Mano y Artroscopia de Muñeca, Afiliada a la Unidad de Cirugía de Mano, Hospital Beata María Ana, Madrid, España

2 Medyarthros Artroscopia y Medicina Deportiva, Guadalajara, Jalisco, México

${ }^{3}$ Cirujano Traumatólogo Ortopedista, Especialista en Cirugía de la Mano, Artroscopia de Muñeca y Microcirugía, Unidad de Cirugía de Mano, Hospital Beata María Ana, Madrid, España

${ }^{4}$ Unidad de Cirugía de Mano, Hospital VITHAS Internacional, Madrid, España

${ }^{5}$ Cirujano Traumatólogo Ortopedista, Especialista en Cirugía de la Mano, Director de la Clínica de Mano, Centro de Artroscopia y Medicina Deportiva Medyarthros, Guadalajara, Jalisco, México
}

\author{
Address for correspondence Raquel A. Montes-Pérez, MD, Cirujana \\ Traumatóloga Ortopedista, Fellowship en Cirugía de la Mano y \\ Artroscopia de Muñeca, Afiliada a la Unidad de Cirugía de Mano, \\ Hospital Beata María Ana, Madrid, España \\ (e-mail: draraquelamontes@gmail.com).
}

\section{Resumen}

\section{Palabras clave}

- dupuytren

- injerto

- piel de espesor total
Introducción No existe un algoritmo de manejo estándar de la recurrencia de la Enfermedad de Dupuytren; el injerto de piel de espesor total posterior a dermofasciectomía ofrece una opción viable para el manejo. El objetivo del estudio fue describir el tamaño del injerto, movilidad articular, satisfacción con la apariencia y funcionalidad, así como complicaciones en pacientes con recurrencia de Enfermedad de Dupuytren, tratados con injerto de piel de espesor total posterior a dermofasciectomía.

Material y Métodos Se realizó un estudio de cohorte en pacientes con recurrencia de Enfermedad de Dupuytren tratados con injerto de espesor total de piel posterior a la dermofasciectomía. Se incluyeron 22 dedos de 19 pacientes con edad promedio de 64,7 años. Las principales variables registradas fueron: grados de flexión pre y postquirúrgica en la articulación MF e IFP, tamaño del injerto, tiempo de seguimiento postquirúrgico, satisfacción de los resultados con la apariencia del injerto y funcionalidad en la vida diaria, así como las complicaciones.

Resultados El tamaño promedio del injerto fue de $3,3 \times 1,7 \mathrm{~cm}$ y la duración del seguimiento de 24,7 meses en promedio. En el postquirúrgico, se alcanzó extensión completa de la MF; encontraste con $55,7^{\circ}$ de flexión en el pre quirúrgico. La articulación IFP presentó, en promedio, flexión de $64,2^{\circ}$ (pre-quirúrgico) y en el postquirúrgico, se logró flexión y extensión completa en 8 dedos; déficit de extensión de $25^{\circ}$ en 6 y contractura de $78,7^{\circ}$, en promedio, en 8 dedos. Diez de 19 pacientes, dijeron estar muy satisfechos con la apariencia del injerto y funcionalidad. Las complicaciones más frecuentes fueron alteraciones sensitivas. received

January 21, 2019

accepted

August 26, 2019
DOI https://doi.org/

10.1055/s-0039-1698791. ISSN 1698-8396.
Copyright $\odot 2019$ Thieme Revinter

Publicações Ltda, Rio de Janeiro, Brazil
License terms

(c) (i) $\ominus$ (\$) 


\begin{abstract}
Abtract

\section{Keywords}

- dupuytren

- grafting

- full-thickness skin

Introduction There is no standard management algorithm for recurrence of Dupuytren's disease; the full-thickness skin graft after dermophasciectomy offers a viable option for its management. The objective of the study was to describe graft size, joint mobility, personal satisfaction with appearance and functionality, as well as, complications in patients with recurrence of Dupuytren's disease treated with fullthickness skin graft after dermophasciectomy.

Material and Methods A cohort study was performed in patients with recurrence of Dupuytren's disease treated with full-thickness skin graft after dermophasciectomy. We included 22 fingers of 19 patients with average age of 64.7 years. The main variables recorded were: pre and postoperative flexion grades in the MCP and PIP joint, graft size, postoperative follow-up time, personal satisfaction of the results, with graft appearance and daily life functionality, as well as complications.

Results 19 patients were included, all of them improved range of movement and functionality. Personal satisfaction based on aesthetics and mobility was classify as "nothing, little and very satisfied". Seventeen patients referred very and little satisfied. Two patients were not, due to the appearance and the little mobility obtained with the graft. The most reported complication was hyperesthesia.

Conclusion Good results were demonstrated in degrees of mobility and satisfaction with regard to appearance and functionality; there are no previous reports with description of the graft size used. In our experience, graft placement is an adequate therapeutic option.
\end{abstract}

Conclusión Se demostraron buenos resultados en los grados de movilidad y satisfacción respecto a la apariencia y funcionalidad; no existen reportes previos con la descripción del tamaño del injerto utilizado. En nuestra experiencia, la colocación de injerto es una opción terapéutica adecuada.

\section{Introducción}

La Enfermedad de Dupuytren es una enfermedad inflamatoria crónica del tejido conjuntivo de etiología desconocida ${ }^{1}$; la prevalencia es de $4 \%-6 \%$ en caucásicos. ${ }^{2}$ Su fisiopatología se centra en los miofibroblastos que producen colágeno tipo $3 \mathrm{y}$ haces de miofibrillas que se acortan progresivamente $\mathrm{y}$ conducen a contracturas de los tejidos blandos y articulaciones. $^{3}$ La enfermedad evoluciona en tres fases: proliferativa, involutiva y residual. Es un trastorno familiar de penetrancia variable; hasta la fecha, ningún gen único ha sido identificado y no existen pruebas genéticas especificas. ${ }^{3}$ Aunque existen tratamientos no quirúrgicos de la Enfermedad de Dupuytren, la cirugía ${ }^{1,4,5}$ se considera el pilar del tratamiento con las siguientes opciones: aponeurectomía con aguja, fasciotomía, fasciectomía, dermofasciectomía y eventualmente, amputación. ${ }^{6}$ Sin embargo, ningún procedimiento tiene superioridad absoluta, y la recurrencia es extremadamente variable ( 2 a $86 \%)^{1,5,7}$

En la recurrencia de la Enfermedad de Dupuytren las manifestaciones clínicas pueden ser más severas debido a las cicatrices y distorsión anatómica de procedimientos previos. ${ }^{1}$ Por otro lado, el manejo es un reto para el cirujano debido a que se incrementa la incidencia de lesiones arteriales $(25,7 \%)$ y nerviosas $(17 \%)$; en contraste, en la enfermedad primaria es de $1,7 \%$ y $3,1 \%$, respectivamente. ${ }^{1}$ Desde $2014,{ }^{5}$ la recurrencia se define como déficit de extensión pasiva mayor de $20^{\circ}$ de al menos una de las articulaciones tratadas, en presencia de una cuerda palpable, comparada con el resultado obtenido en el momento 0 (considerado entre 6 semanas y 3 meses postquirúrgicos); no obstante, esta definición, el manejo quirúrgico de la recurrencia, se fundamenta mayormente en la dificultad funcional del dedo afectado y la decisión del tratamiento es compartida entre el paciente y cirujano. $^{2}$

El manejo quirúrgico de la recurrencia de Dupuytren, requiere mayor experiencia quirúrgica en cirugía de la mano, que se hace más exigente con cada recurrencia; una vez que la contractura es liberada, permanecen defectos de cobertura que en ocasiones por sus dimensiones puede ser imposible cubrir en su totalidad; a ese respecto, la efectividad del injerto de piel de espesor total ha sido descrita desde $1969 .{ }^{8}$ En la década de los 90 s, se demostró un mejor control de la enfermedad y apariencia cosmética aceptable con el injerto de piel de espesor total, además de la durabilidad de éste, posterior a dermofasciectomía. ${ }^{9-11}$ No encontramos reportes específicos del tamaño del injerto; probablemente, eso ha desalentado la colocación de injertos de espesor total de piel para el manejo de la recurrencia de Dupuytren. El objetivo del estudio fue describir el tamaño del injerto, movilidad articular, satisfacción personal con la apariencia y funcionalidad, así como, complicaciones en pacientes con recurrencia de Enfermedad de Dupuytren tratados con injerto de piel de espesor total posterior a dermofasciectomía. 


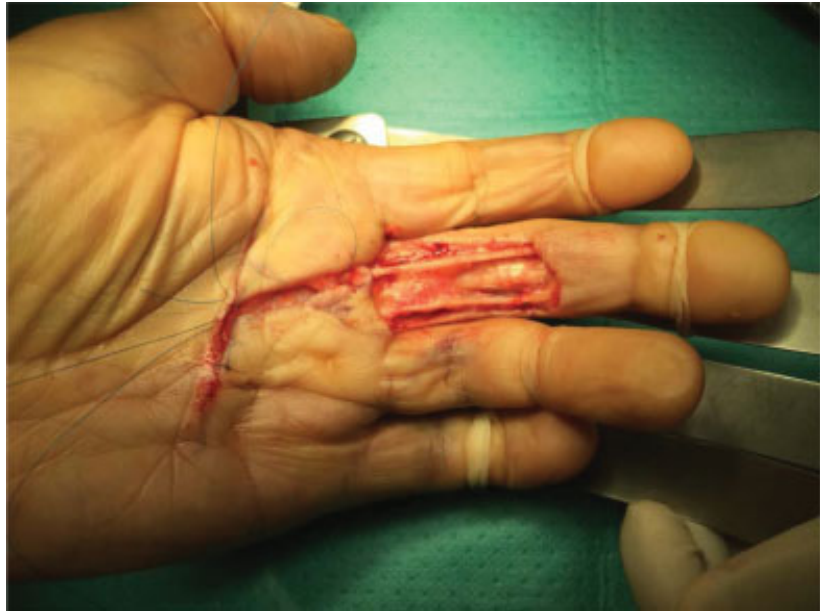

Fig. 1 Diseño cuadrado o rectangular después de retirar la porción de piel y fascia.

\section{Material y Método}

Se realizó un estudio de cohorte de pacientes con diagnóstico de recurrencia de Enfermedad de Dupuytren tratados con injerto de piel de espesor total posterior a dermofasciectomía y antecedente de fasciectomía previa; los casos fueron intervenidos en la Unidad de Cirugía de Mano del Hospital Beata María Ana, de febrero del 2015 a octubre del 2018. Los criterios de inclusión fueron la evidencia de flexo articular metacarpofalángica (MF) o interfalángica proximal (IFP) mayor de $30^{\circ}$, con base en la dificultad funcional del dedo afectado y la decisión del cirujano, así como el antecedente de fasciectomía previa por Enfermedad de Dupuytren en el dedo o dedos afectados por la recurrencia. Se excluyeron los casos con información incompleta en el seguimiento y pacientes con otra patología concomitante de la mano.

Se incluyeron 22 dedos de 19 pacientes con recurrencia de Dupuytren; 13 hombres y 6 mujeres con edad promedio de 64,7 años $(D E \pm 11.4)$ : fueron 12 manos derechas y 7 manos izquierdas. El dedo más afectado fue el 5 to, seguido del 4 to y 2 do dedo; se encontró bilateralidad en 15 de 19 pacientes y cirugías previas en la mano afectada, de 1,3 en promedio (rango 1 a 3). El antecedente familiar positivo, más frecuente, se relacionó con la afectación del padre o hermanos (4y 3 casos, respectivamente); 5 pacientes fumaban y consumían alcohol, dos más, refirieron consumo solo de alcohol y un paciente solamente tabaco. Únicamente, una paciente refirió hipotiroidismo y se encontraba con tratamiento hormonal sustitutivo.
Se registraron las siguientes variables: tiempo de evolución con la Enfermedad de Dupuytren, grados de flexión registrados con goniómetro, antes de la colocación del injerto en la articulación MF e IFP; las mediciones se obtuvieron del expediente clínico; no se registraron, por separado, el déficit de extensión y contractura. Además, se midió el tamaño del injerto en centímetros y áreas anatómicas injertadas (MF, F1 falange proximal, F2 falange media). En el seguimiento posterior al procedimiento quirúrgico, se evaluó movilidad de las articulaciones MF, IFP e interfalángica distal (IFD), el tiempo de recuperación hasta el estado clínico que presentó en la evaluación postquirúrgica, el tiempo de seguimiento desde la realización de la cirugía y las características clínicas del injerto; no se realizó biopsia para identificar la recurrencia de Dupuytren en la piel injertada posterior al procedimiento.

Se preguntó a los pacientes acerca de la satisfacción personal de los resultados con la apariencia del injerto y funcionalidad en la vida diaria con el procedimiento quirúrgico realizado: las respuestas fueron clasificadas de manera cualitativa con la escala tipo Likert con base en los siguientes parámetros: muy satisfecho (3), poco satisfecho (2) o nada satisfecho (1). Además, se indagó la posibilidad, afirmativa o negativa, de volverse a operar conociendo los resultados clínicos al momento de la evaluación. Asimismo, se preguntó sobre la presencia de las siguientes complicaciones: dolor, hiperestesia, hipoestesia o intolerancia al frio.

\section{Procedimiento}

Todos los procedimientos fueron realizados por el mismo cirujano (MDCG). Se realizó bloqueo del plexobraquial con la extremidad en abducción y supinación. Posterior a la colocación de la isquemia en el brazo, se realizó una incisión que incluyó todo el tejido patológico y la piel considerando las áreas, metacarpofalángica e interfalángica proximal de la mano. En la zona afectada, se identificaron y aislaron los paquetes neurovasculares, y se procedió a la dermofasciectomía. Se creó un área de forma rectangular o cuadrada en función de la cantidad de tejido afectado (-Fig. 1) que permitió la medición del injerto requerido (-Fig. 2); éste, se obtuvo de la región antero-interna del brazo ipsilateral. El injerto, se extrajo en forma elíptica y la zona donante se suturó de manera directa (-Fig. 3); a continuación, se desgrasó y se colocó sobre la zona receptora con el tamaño suficiente para cubrirla por completo, realizando sobre el mismo, pequeñas perforaciones para permitir el drenaje del hematoma (-Fig. 4). Posteriormente, se aplicó un apósito
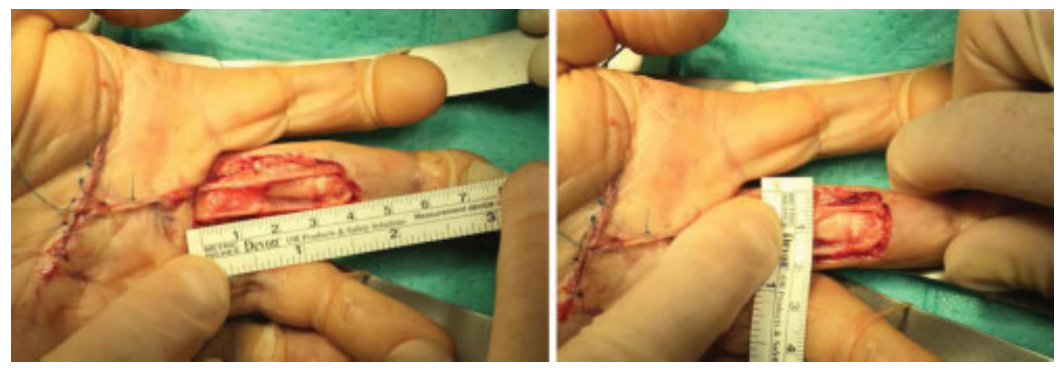

Fig. 2 Medición del área a cubrir posterior a dermofasciectomía en la recurrencia de Dupuytren. 


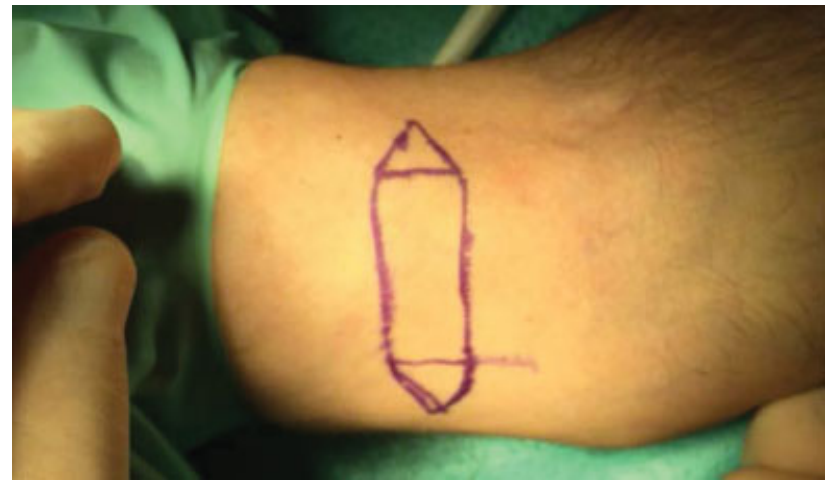

Fig. 3 Brazo ipsilateral, el tamaño del injerto corresponde al área medida.

con gasas para mantener la compresión sobre el lecho donde se colocó el injerto ( Fig. 5). Por último, se utilizó una férula en extensión completa de los dedos para inmovilizar la mano durante dos semanas. El apósito que cubrió el injerto se vigiló periódicamente en consulta y se hidrató continuamente hasta retirarlo. Dos semanas después de la intervención; el paciente inició la movilidad completa, a tolerancia, de la mano y continuó, semanalmente, con vigilancia de la evolución del injerto, retiro progresivo de todas las suturas y movilidad (-Fig. 6). Se continuó con el seguimiento periódico anual.

\section{Análisis Estadístico}

Los datos se registraron en una base de datos con el programa Excel (Microsoft ${ }^{\circledR}$, Seattle WA. EUA). El análisis de los datos se presentó con estadística descriptiva que incluyó: frecuencias, promedios y desviación estándar.

\section{Resultados}

El tiempo promedio de evolución con diagnóstico de la Enfermedad de Dupuytren fue de 11,7 años (DE \pm 9.4$)$. El tamaño del injerto, en promedio, fue de $3,3 \mathrm{~cm}$ de largo por $1,7 \mathrm{~cm}$ de ancho (-Tabla 1); el injerto de mayor tamaño utilizado fue de aproximadamente $5,7 \mathrm{~cm}$ por $4 \mathrm{~cm}$ en un paciente con afectación del 3, 4 y 5 dedo (-Fig. 7). Las áreas anatómicas sobre las que se colocó el injerto fueron MF y F1 en 16 dedos, MF en 4, MF, F1 y F2 en otro caso (dos dedos) (-Tabla 1). Los injertos tuvieron una evolución clínica satisfactoria con integración flexible y estable al esqueleto

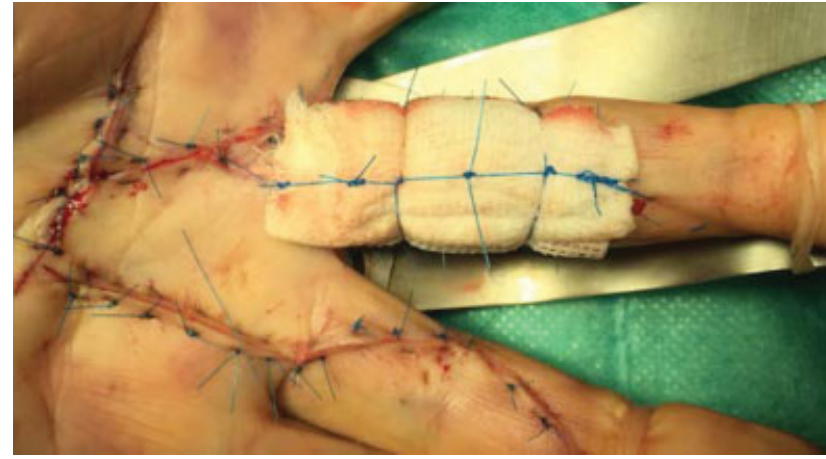

Fig. 5 El apósito consiste en una porción de Linitul, gasa húmeda y gasa seca que se sutura sobre la zona injertada y permanece hasta su retiro, dos semanas después.

digital, sin problemas de ulceración o agrietamiento. El tiempo de recuperación, posterior a la cirugía, hasta la función máxima alcanzada fue en promedio de 3,6 meses (rango 1 a 24); en 16 de 22 dedos, el tiempo de recuperación fue igual o menor a 2 meses: el seguimiento promedio fue de 24,7meses (rango 1 a 45). (-Tabla 1).

En 18 pacientes (21 dedos) se encontró, previo a la cirugía, flexo en promedio de $55,7^{\circ}$ de la MF ( Tabla 1); en el seguimiento posterior a la cirugía, se alcanzó flexoextensión completa: únicamente, un paciente presentó déficit de extensión de $55^{\circ}$ que fue atribuido a cicatriz retráctil, no dolorosa en el borde libre del injerto. Previo a la cirugía, la articulación IFP presentó, en promedio, flexo de $64,2^{\circ}$ ( - Tabla 1); posterior a la cirugía, los resultados se presentan en tres categorías que son flexión y extensión completa (7 pacientes), déficit de extensión ( 5 pacientes) y contractura en flexión ( 7 pacientes): se logró flexión y extensión completa en 8 dedos. En contraste, 6 dedos presentaron déficit de extensión de $25^{\circ}$, en promedio, y 8 dedos presentaron contractura de $78,7^{\circ}$ en promedio. Es importante señalar que dos pacientes (tres dedos), tuvieron el mismo grado de flexión pre y postquirúrgica: mientras que, únicamente un paciente (un dedo), empeoró el grado de flexión (caso no. 3). La articulación IFD, se encontró sin afectación en la evaluación preoperatoria y permaneció sin cambios en el seguimiento posterior a la cirugía en todos los pacientes.

En la - Tabla 2, se presentan los resultados de satisfacción personal con la apariencia y funcionalidad para las actividades diarias, referidas por el paciente, en el seguimiento posterior a la intervención quirúrgica; 10 de
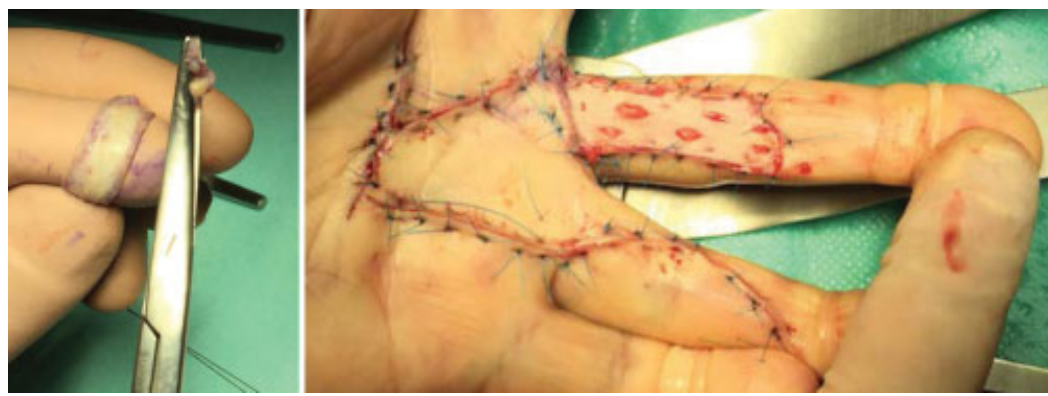

Fig. 4 Extracción de la grasa subcutánea y colocación del injerto de piel de espesor total sobre la zona receptora. 


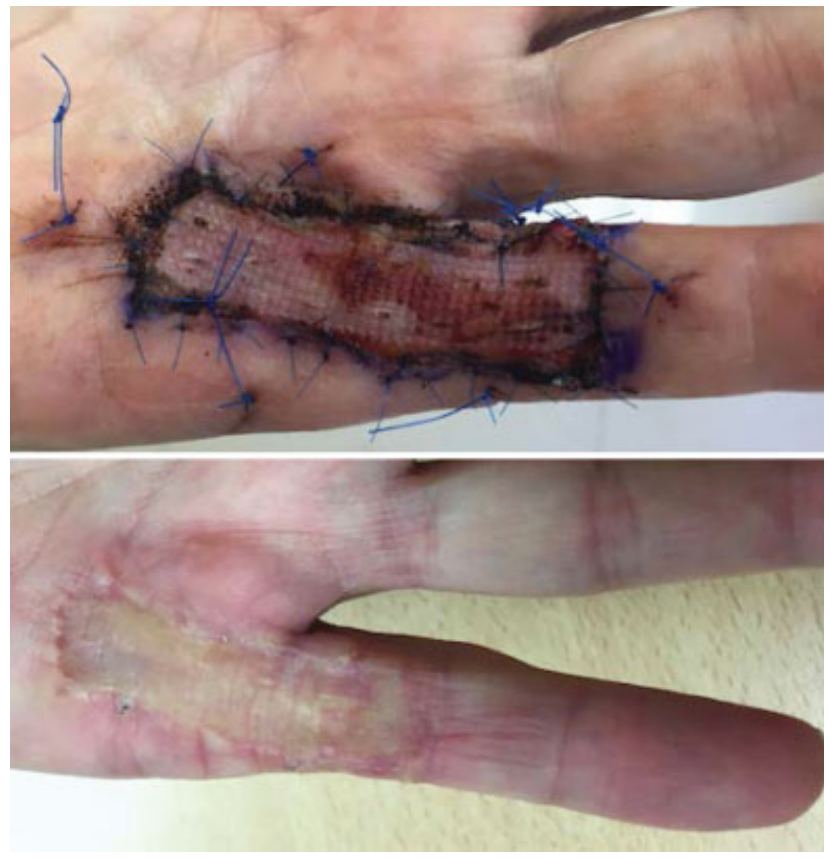

Fig. 6 Retiro progresivo de las suturas, se indica al paciente la movilidad continua a tolerancia.

19 pacientes, se refirieron muy satisfechos; en contraste, 7 de 19, poco satisfechos, debido a que esperaban mayor grado de movilidad. Por otro lado, dos pacientes estuvieron nada satisfechos porque la apariencia del injerto sobre la piel de la palma no fue la deseada y existió poca diferencia en la movilidad obtenida con relación a la mano contralateral, también afectada; ésta, se manejó con fasciectomía sin injerto. A la pregunta: ¿Se volvería a operar conociendo los resultados obtenidos? En 14 de 19 pacientes la respuesta fue afirmativa y únicamente 5 pacientes no volverían a operarse con el referente del resultado logrado. En la - Tabla 3, se describen las complicaciones en 12 de 19 pacientes; las más frecuentes fueron hiper-hipoestesia, 7 pacientes no presentaron ninguna complicación. Un paciente desarrolló cicatriz marginal hipertrófica no dolorosa (-Fig. 8) no se encontraron nódulos o cuerdas sobre los injertos que pudieran relacionarse con infiltración por Dupuytren, sin embargo, no se realizó estudio histológico para demostrarlo.

\section{Discusión}

En nuestro estudio, se utilizaron injertos de piel de espesor total, en promedio, de $3,3 \times 1,7 \mathrm{~cm}$ para la cobertura de las áreas donde se realizó dermofasciectomía en pacientes con recurrencia de Enfermedad de Dupuytren con buenos resultados en los grados de movilidad y satisfacción del paciente con respecto a la apariencia y funcionalidad con seguimiento de hasta 45 meses. No se encontraron reportes previos a nuestro estudio con la descripción del tamaño del injerto utilizado. En nuestra experiencia, la realización de dermofasciectomía y colocación de injerto es una opción terapéutica adecuada.

El injerto de espesor total de piel en la recurrencia de Enfermedad de Dupuytren se propuso como un mecanismo de "cortafuegos" para evitar la progresión y prevenir el desarrollo de la deformidad por contractura. ${ }^{12}$ Nosotros consideramos que, se saben tres cosas con certeza: la imposibilidad de una cura, la progresión que, aunque variable, está presente y que independientemente del manejo, la posibilidad de recurrencia es un escenario común. La bibliografía es vasta en relación con las diferentes alternativas del manejo, en contraste, existe poca información, en años recientes, de la utilización del injerto de piel de espesor total; su uso principal es la cobertura de áreas pequeñas o eligiendo dejarlas al cierre por segunda intención. ${ }^{2}$ Es difícil identificar en la etapa inicial del diagnóstico, los casos con recurrencia, por lo que la conducta quirúrgica de utilizar injerto de piel depende de la preferencia y habilidad del cirujano para tratar la pérdida de sustancia resultante de la eliminación de la piel invadida. $^{13}$ Un estudio realizado por Ullah y col., ${ }^{7}$ compararon el uso de dermofasciectomía más injerto de piel de espesor total como "cortafuegos" y la fasciectomía en 90 dedos, con seguimiento de 36 meses; no se demostró corrección de la contractura o disminución en la recurrencia después de la dermofasciectomía “cortafuegos." Entre los dos grupos de este estudio, se obtuvo corrección completa postquirúrgica de la contractura de la MF; estos resultados son comparables con nuestro estudio; 18 de 19 pacientes ( 21 de 22 dedos) mostraron movilidad completa de esa articulación. En otro estudio, ${ }^{9}$ se reportó contractura en flexión de la IFP de $9^{\circ}$ en promedio de 58 rayos y contractura de $45^{\circ}$ en promedio de 31 rayos. En nuestro estudio, se encontró contractura en promedio de $78,7^{\circ}$ en 8 dedos. Por otro lado, nosotros presentamos tres categorías: flexo extensión completa, déficit de extensión y contractura que puede simplificar la evaluación clínica funcional; por lo que la comparación con otros estudios se limita por esas diferencias metodológicas.

La satisfacción personal con la apariencia del injerto de espesor total posterior a dermofasciectomía en recurrencia de Dupuytren, ha sido publicada previamente. ${ }^{9-11}$ En nuestro estudio, además de la apariencia, se evaluó la funcionalidad para las actividades diarias; 10 de 19 pacientes, se encontraron muy satisfechos: únicamente dos pacientes estuvieron nada satisfechos con los resultados postquirúrgicos: nosotros consideramos que se debe lograr, además de la apariencia aceptable para el paciente, la funcionalidad de la mano y, eventualmente, la aceptación de un procedimiento quirúrgico futuro. Por otro lado, las complicaciones en el manejo de la recurrencia de Enfermedad de Dupuytren son más frecuentes; específicamente las lesiones nerviosas y arteriales. ${ }^{1}$ En nuestro estudio, las complicaciones fueron alteraciones sensitivas que ocurrieron en 12 de 19 pacientes; ningún paciente reportó anestesia distal o lesiones vasculares. Además, se ha reportado recurrencia con infiltración histológica del tejido injertado ${ }^{14}$; en nuestro estudio con seguimiento de 24,7 meses en promedio, no encontramos clínicamente recidivas sobre la piel del injerto.

Las fortalezas del estudio se relacionan con el procedimiento quirúrgico realizado por el mismo cirujano y la descripción del tamaño del injerto de espesor total de 


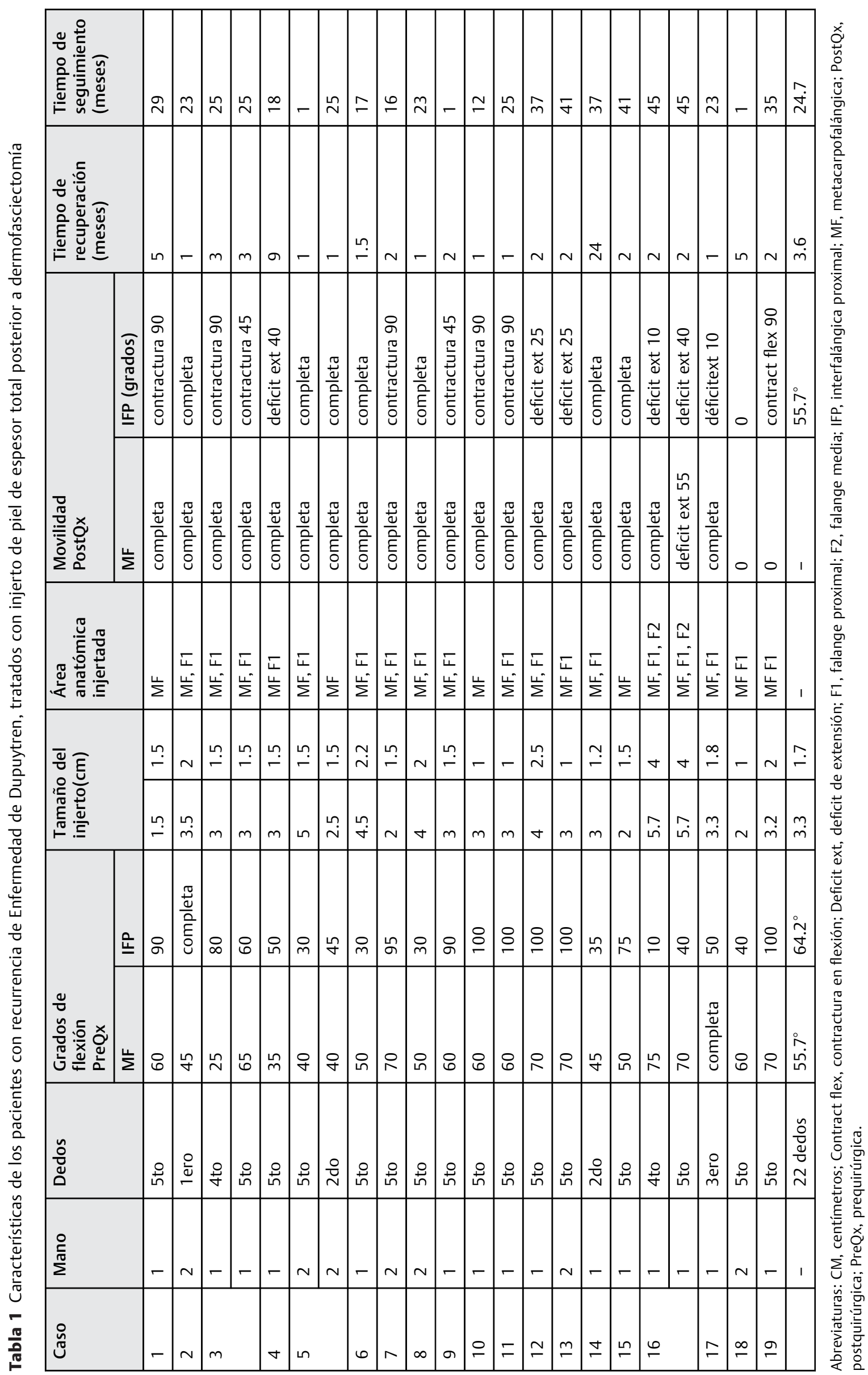




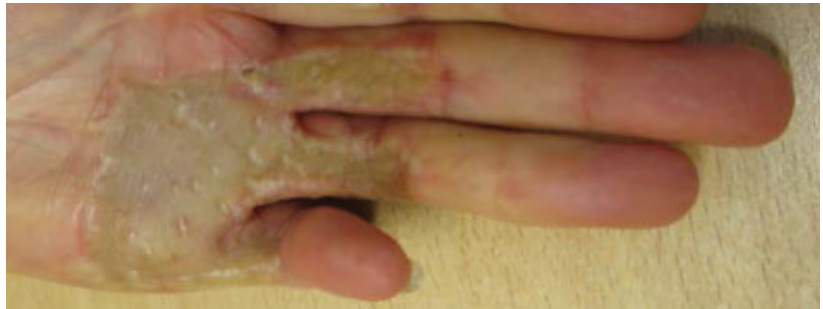

Fig. 7 Injerto de mayor tamaño, abarca las áreas anatómicas MF, F1 y F2.

Tabla 2 Satisfacción personal con la apariencia y funcionalidad para las actividades diarias de pacientes con recurrencia de Enfermedad de Dupuytren, tratados con injerto de piel de espesor total posterior a dermofasciectomía

\begin{tabular}{|l|l|}
\hline Apariencia y funcionalidad & $\begin{array}{l}\mathbf{n}=19 \\
\text { (22 dedos) }\end{array}$ \\
\hline Nada satisfecho & 2 \\
\hline Poco satisfecho & 7 \\
\hline Muy satisfecho & 10 \\
\hline Si se volvería a operar & 14 \\
\hline
\end{tabular}

Tabla 3 Complicaciones de los pacientes con recurrencia de Enfermedad de Dupuytren, tratados con injerto de piel de espesor total posterior a dermofasciectomía

\begin{tabular}{|l|l|}
\hline Variable & $\begin{array}{l}\mathbf{n = 1 9} \\
(22 \text { dedos) }\end{array}$ \\
\hline Ninguna & 7 \\
\hline Hiperestesia & 3 \\
\hline Hipoestesia & 4 \\
\hline Intolerancia al frio & 2 \\
\hline Dolor e Hiperestesia & 1 \\
\hline Dolor e Hipoestesia & 1 \\
\hline $\begin{array}{l}\text { Hipoestesia, hiperestesia e } \\
\text { intolerancia al frío }\end{array}$ & 1 \\
\hline
\end{tabular}

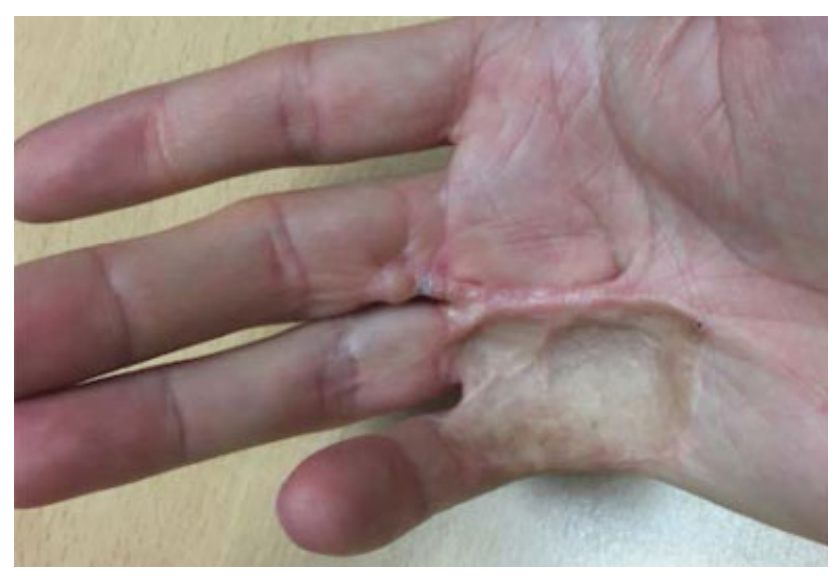

Fig. 8 Cicatriz hipertrófica en el borde libre del tejido injertado. piel, utilizado en un grupo homogéneo de pacientes con recurrencia de Dupuytren; otra fortaleza es la evaluación conjunta de la satisfacción con apariencia y funcionalidad para las actividades de la vida diaria. Las limitaciones se relacionan con el diseño retrospectivo del estudio, el tamaño de muestra pequeño y no se evaluó la Movilidad Activa Total prequirúrgica y postquirúrgica. Por otro lado, no se realizó biopsia para identificar infiltración de Enfermedad de Dupuytren sobre el injerto o en sus bordes libres y la duración del seguimiento es insuficiente para determinar la evolución a largo plazo.

\section{Conclusión}

En nuestro estudio, se demostraron buenos resultados de movilidad y satisfacción en la apariencia y funcionalidad para actividades de la vida diaria de los pacientes con recidiva de Enfermedad de Dupuytren que fueron tratados con dermofasciectomía y colocación de injerto de espesor total en el seguimiento promedio de 24,7 meses. En nuestra experiencia, este procedimiento ofrece resultados efectivos; se requieren estudios clínicos prospectivos y a largo plazo para establecer conclusiones definitivas e incentivar esta modalidad de manejo.

\section{Conflicto de Intereses}

Los autores del trabajo declaran no tener ningún conflicto de intereses.

\section{Agradecimientos}

Los autores agradecen al doctor Rafael Ortega Orozco director del Centro de Medicina Deportiva y Artroscopia MEDYARTHROS y a la profesora Ana María Contreras Navarro de la Unidad de Investigación y Desarrollo Tecnológico (UNIDET) por su apoyo en la realización de este articulo.

\section{Bibliografía}

1 Denkler K. Surgical complications associated with fasciectomy for dupuytren's disease: a 20-year review of the English literature. Eplasty 2010;10:e15

2 Dias JJ, Aziz S. Fasciectomy for Dupuytren Contracture. Hand Clin 2018;34(03):351-366

3 Zhang AY, Kargel JS. The Basic Science of Dupuytren Disease. Hand Clin 2018;34(03):301-305

4 Werker PMN, Pess GM, van Rijssen AL, Denkler K. Correction of contracture and recurrence rates of Dupuytren contracture following invasive treatment: the importance of clear definitions. JHand Surg Am 2012;37(10):2095-2105.e7

5 Felici N, Marcoccio I, Giunta R, et al. Dupuytren contracture recurrence project: reaching consensus on a definition of recurrence. Handchir Mikrochir Plast Chir 2014;46(06):350-354

6 Kaplan FTD, Crosby NE. Treatment of recurrent dupuytren disease. Hand Clin 2018;34(03):403-415

7 Ullah AS, Dias JJ, Bhowal B. Does a 'firebreak' full-thickness skin graft prevent recurrence after surgery for Dupuytren's contracture?: a prospective, randomised trial JBone Joint Surg Br 2009;91(03): 374-378

8 Heuston JT. The control of recurrent Dupuytren's contracture by skin replacement. Br J Plast Surg 1969;22(02):152-156 
104 Injerto de Piel de Espesor Total en Recurrencia de Dupuytren Montes-Pérez y col

9 Hall PN, Fitzgerald A, Sterne GD, Logan AM. Skin replacement in Dupuytren's disease. J Hand Surg [Br] 1997;22(02):193-197

10 Brotherston TM, Balakrishnan C, Milner RH, Brown HG. Long term follow-up of dermofasciectomy for Dupuytren's contracture. Br J Plast Surg 1994;47(06):440-443

11 Searle AE, Logan AM. A mid-term review of the results of dermofasciectomy for Dupuytren's disease. Ann Chir Main Memb Super 1992;11(05):375-380
12 Hueston JT. 'Firebreak' grafts in Dupuytren's contracture. Aust N Z J Surg 1984;54(03):277-281

13 Villani F, Choughri H, Pelissier P. Intérêt de la greffe de peau dans la prévention des récidives de la maladie de Dupuytren. Chir Main 2009;28(06):349-351

14 Wade RG, Igali L, Figus A. Dupuytren disease infiltrating a full-thickness skin graft. JHand Surg Am 2016;41(08): e235-e238 